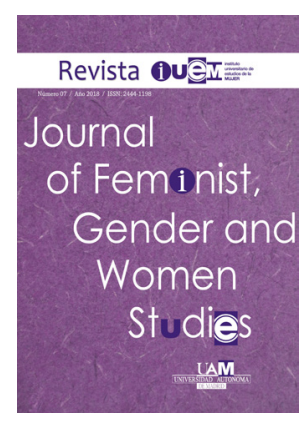

\section{Mujeres, diversidad funcional y multidiscriminación}

\author{
Women, functional diversity and multiple discrimination \\ Sandra Candelas Tejada ${ }^{1, @ y ~ G r a c i e l a ~ M u l e t ~ L o ́ p e z ~}{ }^{2, @ ~}$ \\ ${ }^{1}$ Equipo Andecha, Participación y Trabajo Comunitario. \\ ${ }^{2}$ Asociación OjosqueOyenEspaña. \\ @ Autor/a de correspondencia: sandra.candelas@equipoandecha.org, gracielamulelopez@gmail.com
}

\title{
Resumen
}

Las violencias ejercidas contra las mujeres leídas como discapacitadas están tan naturalizadas en la sociedad que son una de las formas de discriminación menos visibles. Esta naturalización es fruto de un sistema de opresiones que tienen su origen en el hecho de ser mujeres y de ser leídas como discapacitadas, y por tanto, en una norma social sexista y capacitista. A través de la creación de un grupo motor de mujeres con diversidad funcional y la aplicación de instrumentos del diagnóstico participativo facilitamos un espacio formativo en el que: se visibilizó que la discapacidad cosifica a las mujeres y las somete a múltiples violencias encadenadas, naturalizadas y, por tanto, invisibilizadas; que esta situación las lleva a vivir en un estado permanente de tensión psicológica y física que pone a prueba sus recursos sociales y habilidades personales y orienta las soluciones hacia el miedo y la culpa; y que para abordar estas problemáticas se ha de tener en cuenta un enfoque psicosocial que permita a las mujeres salir de la situación de víctimas e incorpore los principios de reconocimiento, reparación y no repetición. Como conclusión al trabajo, se presentan una serie de estrategias individuales, colectivas e institucionales basadas en las propuestas de la Diversidad Funcional.

Palabras clave: Violencia, mujeres leídas como discapacitadas, discriminación, interseccionalidades, diagnóstico participativo, diversidad funcional.

\begin{abstract}
The many types of violence perpetrated against women who are perceived as being disabled is so naturalized in society that it has become one of the least visible types of discrimination. This naturalization is the fruit of a system of oppression that has its origins in the fact that these people are women and that they are perceived as being disabled, therefore, it is based on a sexist and ableist norm. Thorough the creation of a group of women with functional diversity as the driving force and applying various techniques based on participatory appraisal, we have created an educational space which gave visibility to the fact that disability reify women and brought them under multiple forms of violence, which are naturalized and make them invisible. This situation lead them to live in a state of permanent psychological and physical tension which constantly tests their social resources and personal abilities and orientates solutions towards fear and guiltiness. In order to tackle these problems is necessary to consider a psycho-social approach that allows women to get out of a victim situation and that incorporates principles of recognition, reparation and non-recidivism. As a conclusion, we present a number of individual, collective and institutional strategies based on proposals of Functional Diversity.
\end{abstract}

Keywords: Violence, women who are perceived as being disabled, discrimination, inter-sectional categories, participatory appraisal, functional diversity. 


\section{INTRODUCCIÓN}

“¿Te has preguntado alguna vez si las mujeres leídas como discapacitadas sufrimos violencias machistas?". Con esta pregunta comenzaba el proyecto "Miradas: mujeres, diversidad funcional y multidiscriminación". Nuestro objetivo era visibilizar las numerosas violencias contra las mujeres leídas como discapacitadas, que son invisibilizadas y naturalizadas por la sociedad e incluso aceptadas y/o legitimadas por los estamentos judiciales y administrativos. En este proyecto incorporamos un enfoque interseccional y comprobamos cómo las violencias incluyen múltiples agresiones por el mero hecho de pertenecer a un grupo social diferente, tradicionalmente considerado menos capaz o incapaz y alejado de la "normalidad" (Rodríguez y Ferreira, 2010). ¿YY por qué abordar de forma específica las violencias en mujeres leídas como discapacitadas? Aun sabiendo que las violencias contra cualquier mujer comparten muchos elementos culturales, las violencias contra mujeres leídas como discapacitadas tienen unas características propias [Red Internacional de Mujeres con Discapacidad (INWWD), 2010] derivadas de un modelo de desarrollo que organiza la sociedad de manera segregada en función del binomio "normalidad-anormalidad" (García-Santesmases, 2015). Esta estructuración de los grupos sociales supone que a cada uno de los miembros de esos grupos se le atribuyan una serie capacidades o incapacidades, así como roles, desde un punto de vista productivo, y por lo tanto, económico (Barnes, 2005).

Con el objetivo de visibilizar estas etiquetas negativas e incapacitantes, que son naturalizadas en el contexto cotidiano, a lo largo del artículo se presentarán tres formas diferentes de referirse a las mujeres con funcionalidades diversas.

- Mujer con discapacidad o discapacitada ${ }^{1}$. Término peyorativo que etiqueta, reduce y cosifica a las personas mediante la asignación de una cualidad o atributo que prescribe un estándar social de normalidad y que incorpora una serie de roles esperados para las mujeres. Responde a la necesidad de mantener la desigualdad social y los privilegios de un colectivo privando a otra parte de la sociedad de los mismos. Término utilizado de forma naturalizada por el conjunto de la sociedad que no deja lugar a las capacidades y habilidades de las personas y que invisibiliza o castiga la experiencia de la diversidad.

- Mujer leída como discapacitada. Término nuevo, acuñado por el proyecto Miradas, y que incorpora un enfoque psicosocial y feminista a la discapacidad. Dado que la sociedad está organizada en torno a una serie de estratos y grupos sociales, es la sociedad la que lee (organiza) a las personas de acuerdo al binarismo capacidad (normalidad)discapacidad (anormalidad). Hace referencia a cómo somos percibidas socialmente, poniendo el énfasis o evidenciando los procesos de multidiscriminación y cosificación que experimenta un colectivo y que son silenciados social e institucionalmente.

- Mujer con diversidad funcional (DF). Término propuesto por el Foro de Vida Independiente ${ }^{2}$ (Romañach

1 Para quien quiera profundizar en la evolución del concepto de discapacidad, puede consultar Shum et al. (2006).

2 El Foro de Vida Independiente y Divertad tiene su origen a mediados del y Lobato, 2005), que incorpora el enfoque de la diversidad. No es sinónimo de discapacidad, ni un concepto alternativo o eufemismo, tampoco es una palabra políticamente correcta. Este concepto pone el foco en las diversas formas que tenemos de realizar diferentes actividades. Nombra de forma positiva a un grupo de personas tradicionalmente excluidas y estigmatizadas, propiciando su inclusión en la sociedad de una forma activa. Pero no solo eso, además crea una categoría social nueva que beneficia al conjunto de la sociedad, al reconocer positivamente la diversidad. Dignidad, respeto y derechos fundamentales son la base de esta manera de nombrar.

\section{MÉTODO}

\section{Participantes}

El grupo motor estaba formado por 16 mujeres, 13 de las cuales tenían asignada algún tipo de discapacidad (auditiva, intelectual, psíquica, motora, orgánica y física) y tres no la tenían asignada. Los criterios de selección fueron la lectura de discapacidad (reservándose un 10\% de las plazas a mujeres no leídas como discapacitadas) y la pertenencia a organizaciones que trabajasen temas de DF, género y feminismos. Las vías de difusión para la creación del grupo fueron la articulación con recursos comunitarios de los distritos y la difusión por redes sociales.

\section{Método}

Se diseñó un programa de formación de promotoras comunitarias en multidiscriminación, género y DF organizado en torno a 20 sesiones, entre los meses de diciembre de 2016 y junio de 2017. Nos apoyamos en diversas estrategias didácticas de la Educación Popular por su "carácter abierto y flexible, participativo, grupal, práctico y vivencial" (Vargas et al., 1995:8) y en una serie técnicas del diagnóstico comunitario que permitieron recoger testimonios en primera persona y crear un saber colectivo en materia de discapacidad, diversidad funcional, violencias, multidiscriminación, participación y desarrollo comunitario. También se incorporaron en las sesiones apoyos técnicos y humanos que facilitaron la participación plena de un grupo diverso funcionalmente (intérpretes, mediadoras, asistentes personales y materiales de lectura fácil).

\section{Técnicas}

Se diseñaron y seleccionaron los siguientes instrumentos de análisis y recogida de información:

- Un cuestionario sobre "Percepción social de la discapacidad" para identificar la percepción social que se tiene sobre la discapacidad y la DF, así como sobre la participación social de las mujeres leídas como discapacitadas.

- Creación colectiva de un cómic sobre la experiencia de discapacidad para saber cómo se adaptan las mujeres con DF a la sociedad en la que viven, cómo influyen en la

año 2001 con el objetivo de impulsar en España el Movimiento de Vida Independiente, surgido en EE.UU en 1972 y muy arraigado en Europa en la actualidad. http://forovidaindependiente.org 
construcción de su identidad y la narración que hacen de su experiencia de la discapacidad.

- Registro de testimonios que recogen diferentes experiencias de violencias y multidiscriminación por razones de género y discapacidad, así como las estrategias que las mujeres ponen en marcha para afrontarlas.

- Un recorrido por el distrito con el objetivo de explorar la percepción sensorial que tienen las mujeres con DF sobre el entorno urbanístico, señalando las principales barreras que encuentran y su impacto.

- Una mesa de experiencias sobre multidiscriminación hacia mujeres por razones de interseccionalidad, así como las maneras de afrontarlo individual y grupalmente.

- Elaboración de un sociograma con el que identificar los principales agentes vinculados con la multidiscriminación hacia mujeres leídas como discapacitadas, a través del cual analizar la posición que ocupa cada uno y las relaciones entre ellos.

- Participación y organización de seminarios y jornadas con el fin de implicar a un conjunto de actores sociales en el análisis de la multidiscriminación hacia mujeres leídas como discapacitadas, fomentando el debate y el contraste de opiniones o enfoques.

\section{DISCUSIÓN}

\section{¿Qué es la discapacidad?}

Pese a las numerosas revisiones que se han hecho del concepto (Díaz, 2009), nos seguimos encontrando que la mayor parte de las respuestas reflejan que en el imaginario social perdura la idea de discapacidad como algo negativo, indeseable y limitante (Etxebarria, 2008). Se entiende la discapacidad como una tragedia, un defecto, una desviación o desventaja que hay que tapar, curar, rehabilitar o integrar en la sociedad ${ }^{3}$.

La primera causa de esta respuesta la encontramos en el contexto social, organizado en torno a indicadores de desarrollo centrados, casi exclusivamente, en lo económico y en la capacidad de producción (o de gasto) de las personas. Estos indicadores son asumidos en el seno de una sociedad porque ofrecen una aparente coherencia, consistencia y orden a la vida. Su existencia permite regular las oportunidades, derechos o recursos para cada sujeto y grupo social concreto a través de un sistema de recompensas por las que los miembros de una sociedad compiten y que sostienen una estructura social basada en la estratificación y, por tanto, en la pobreza y la desigualdad (Sen, 2000). Otra de las consecuencias directas de la estratificación es la cosificación (Goffman, 2012) de las personas o colectivos que ocupan los márgenes de la sociedad, es decir, de quienes se desvían de la norma esperada socialmente ${ }^{4}$. La

3 Esta cuestión queda también recogida en los resultados obtenidos en el cuestionario sobre "Percepción de discapacidad" a partir de una muestra de 397 personas leídas y no leídas como discapacitadas, que se realizó durante el proyecto. Para la mayor parte de las personas, la discapacidad era una deficiencia o disminución física, sensorial o psíquica, un impedimento, limitación o falta de capacidad, la consecuencia de una enfermedad, no poder valerse por sí mismo o un sinónimo de minusvalía o invalidez.

4 Las personas leídas como discapacitadas, al no responder al estándar cosificación es un tipo de violencia directa y estructural que acaba configurando: a) las políticas, programas y acciones de las que son destinatarias las personas leídas como discapacitadas (asistencialistas y rehabilitadoras); b) los comportamientos, actitudes y morales predominantes en la sociedad (clasismo, racismo, sexismo, capacitismo, entre otros) y c) las posibilidades de participación en diferentes ámbitos (laboral, relacional, educativo, cultural, etc.). Ejemplos de todo esto lo encontramos en los testimonios de las mujeres recogidos a lo largo del proyecto: "Cuando le dije a mi madre que me quería casar, ella me dijo: '¿Para qué vas a casarte, si no puedes realizar las tareas de la casa porque vas en silla de ruedas?', 'iqué disgusto!'” (testimonio de L.D. 21 de diciembre de 2016); "Percibo que la gente me mira haciendo 'un juicio negativo' sobre mí, y eso hace que me mueva con miedo o con sentimiento de vergüenza en algunos sitios, como, por ejemplo, cuando entro en un restaurante o en una tienda de ropa" (testimonio de E.S. 21 de diciembre de 2016).

La naturalización de la cosificación permite que la sociedad en su conjunto (familiares, profesionales, asociaciones, poderes públicos) considere que la discapacidad es un problema individual (Oliver, 2008) y que la responsabilidad de eliminar las numerosas barreras y violencias ha de recaer sobre las mujeres leídas como discapacitadas en un contexto social que, en vez de ser promotor de solidaridad y apoyo comunitario, considera normal infantilizarlas, minusvalorarlas, temerlas, evitarlas y abusar de ellas. Los testimonios recogidos de las mujeres ponen esto de manifiesto: "Me dijo que la culpa era mía porque yo le provocaba, y que era mejor que no se lo contara nada a nadie" (testimonio C.P. 18 de enero de 2017); "Me dijo que la culpa era mía porque era una inútil" (S.G. 11 de enero de 2017), "Me dijo que no me quejara porque ya recibía muchas ayudas" (testimonio E.S. 18 de enero de 2017), "Me dijo que las personas como yo nunca podrían vivir de manera independiente" (testimonio de T.F. 18 de enero de 2017). En la tabla 1 presentamos la definición de discapacidad creada por el grupo motor.

Como decíamos, la ausencia de apoyos efectivos y el modelo predominante de discapacidad hace que, para la mayor parte de las mujeres consultadas, sea una tarea difícil o casi imposible narrar la violencia y sus impactos, ya que supondría volver a entrar en el ciclo de discriminación y cosificación. Las causas que identificamos se presentan en la tabla 2.

A la hora de responder a estas violencias, también comprobamos que no todas las mujeres reaccionan de la misma manera. Sean las reacciones de un tipo u otro, siempre son síntomas normales frente a situaciones anormales de violencia sobre su persona y entorno más cercano (Martín Beristain y Riera, 2003). En los testimonios recogidos las mujeres expresaban que esta situación les lleva a vivir en un permanente estado de tensión psicológica y física que pone a prueba sus recursos y habilidades personales y orienta las soluciones o estrategias para afrontarlo hacia el miedo,

de normalidad, son valoradas como cosas, como objetos "sin moral, sin alma, desviados, incapaces de definir nuestras necesidades, incapaces de valernos por nosotros mismos, improductivos, desechables, etc. y tratados desde un enfoque de dependencia y 'minusvaloración paternalista'". (Etxebarría, 2008: 35). 


\section{¿Qué es la discapacidad? (una definición desde el proyecto "Miradas")}

Un término:

- Negativo, una barrera impuesta por la sociedad.

- Al que se le atribuyen causas médicas o morales (un castigo divino o una enfermedad).

- Que lleva asociadas numerosas expresiones peyorativas: minusvalía, anormalidad, desviación, incapacidad, retraso, etc.

- Que limita las capacidades, oportunidades y deseos.

- Que destina a las personas a recursos específicos y "especiales", en definitiva, a procesos de institucionalización, segregación, aislamiento, soledad,...

- Que supone una forma de trato poco digna, basada en el sometimiento, la subordinación y la opresión (infantilización, medicalización, etc.).

- Y que recorta o anula nuestra autonomía, autodeterminación y la defensa de nuestros derechos.

Tabla 1: ¿Qué es la discapacidad?

\section{Causas por las que no se identifican los impactos o no se denuncia la multidiscriminación por discapacidad}

- Un contexto social, político y económico que naturaliza o normaliza un sistema de relaciones basado en la violencia (dependencia) que lleva a minimizar o relativizar las denuncias o a responsabilizar a la mujer (generando culpa y sumisión) - Un sentimiento de miedo a las consecuencias de hablar o denunciar: insultos, juicios o malos tratos físicos.

- Preferir olvidar o guardar silencio como estrategia de supervivencia o superación del hecho violento y doloroso.

- Pensar que no va a servir para nada, por falta de confianza en el sistema de ayuda (legal, social, etc.), o porque el sistema de ayuda lo hace muy difícil.

- Por la dificultad de entender lo que ha sucedido y narrarlo.

- Por falta de información sobre derechos y libertades.

- Por producirse en contextos donde es imposible escapar (familia, recursos asistenciales, etc.) o protegerse.

- Porque prevalece la impunidad frente a los espacios de apoyo, reparación y reconocimiento social.

Tabla 2: Causas por las que no se denuncian las violencias ejercidas contra las mujeres leídas como discapacitadas.

la culpa o el aislamiento: "Mi madre me culpa de no tener vida propia, me lo recuerda constantemente. Pero luego es incapaz de dejarme salir a la calle con mis amigas o dejarme un rato sola en casa. Esto provocaba numerosas discusiones que deterioraban la relación familiar" (testimonio de C.P. 25 de enero de 2017). "En los centros educativos especiales los tiempos de recreo son más cortos que en los ordinarios. Algunos profesores nos comentan que es porque tardan más tiempo en meternos en clase y así no se pierde tiempo. Nosotras nos resistíamos y a veces intentábamos evitar que nos movieran" (testimonio E.S. 25 de enero de 2017). "Cuando voy por la calle me enfrento a numerosas barreras (agujeros en el suelo, obras, escalones para acceder a locales, bordillos altos, miradas curiosas de la gente...) que no me permiten disfrutar del paseo o caminar tranquila. Ahora cada vez salgo menos de casa" (testimonio L.G. 25 de enero de2017).

\section{Tipos de impacto que genera la discapacidad}

\section{Degradación de la identidad}

La identidad es un aspecto clave para entender muchos de los efectos de la violencia y la multidiscriminación (Martín Beristain y Riera, 2003). La identidad ayuda a mantener la seguridad emocional y la capacidad de acción o afrontamiento, mediante la toma de conciencia de las situaciones que experimenta una persona, la vivencia de ser uno mismo y el sentimiento de pertenencia a un grupo o comunidad. Si el concepto social de discapacidad es peyorativo y cosificador, la socialización de las mujeres leídas como discapacitadas deposita un conjunto de creencias falsas y destructivas sobre su identidad individual y social muy alejadas de sus propios deseos y capacidades o de la posibilidad de pertenecer a diferentes grupos sociales tal y como vemos en la tabla 3.

Si es en el entorno familiar o en el medio más cercano donde las mujeres leídas como discapacitadas encuentran la mayor parte de los apoyos, no podemos hablar de una sola víctima ${ }^{5}$ ni podemos perder de vista los impactos que recaen sobre dicho entorno: crisis familiares, inhibición de la comunicación, agotamiento o "quemazón" de las redes, aparición de sentimientos de disgusto, odio o culpabilización. En palabras de las propias mujeres del grupo motor: "La ausencia de recursos públicos o privados, así como las escasas redes de apoyo suponen para mi madre que su vida gire en torno a mí. Tiene dificultades para encontrar trabajo, para establecer relaciones de pareja, desarrollar sus aficiones " (testimonio de E.S. 8 de febrero de 2017). "Mi padre se fue de casa porque no podía soportar tener una hija con discapacidad" (testimonio de L.G. 8 de febrero de 2017). "No salgo todo lo que quiero de casa, porque no quiero quemar a las pocas personas que me ofrecen apoyo. Siento que se esfuerzan demasiado" (testimonio de C.B. 8 de febrero de 2017).

\section{Desmovilización colectiva}

Los impactos individuales de la discapacidad impiden

\footnotetext{
5 Entendemos por víctima "toda persona que haya sufrido daños, individual o colectivamente, incluidas lesiones físicas o mentales, sufrimiento emocional, pérdidas económicas o menoscabo sustancial de sus derechos fundamentales, como consecuencia de acciones $u$ omisiones que constituyan una violación manifiesta de las normas internacionales de derechos humanos o una violación grave del derecho internacional humanitario" (ONU, 2005).
} 


\section{Impactos sobre la persona (testimonios recogidos los días 18 y 25 de enero de 2017)}

- Una vida basada en la represión, el ocultamiento o la inhibición: “Voy obligada a este centro" (testimonio de C.R. del18 de enero de 2017).

- Miedo y vergüenza: “No quiero que se rían de mi madre por tener una hija así" (testimonio de C.P. del 18 de enero de 2017). - Baja autoestima: “No sé si seré capaz de hacer este curso" (testimonio de S.B. del 21 de enero de 2017).

- Sentimiento de culpa: “No tenía que haber dejado entrar a mi pareja en la habitación si en la residencia está prohibido, es normal que me regañen" (testimonio de G.D. del 18 de enero de 2017).

- Inhibición de la participación social: "Prefiero no asistir a las actividades programadas por el centro " (testimonio de T.R. del 21 de enero de 2017).

- Evitación: "Tengo miedo de la reacción de mi hermano, así que no me muevo porque pienso que así no me va a tocar" (testimonio de L.G. del 18 de enero de 2017).

- Privatización del daño: "Mejor que nadie sepa lo que me va a pasar, porque van a desconfiar" (testimonio de C.R. del 21 de enero de 2017).

- Búsqueda de estímulos que confirmen las hipótesis sobre la violencia y la autoridad: "Al final es cierto lo que siempre me dicen, que si salía sola me podía caer con la silla de ruedas" (testimonio de L.G. del 18 de enero de 2017).

- Mentira u ocultamiento como forma de percepción de control o autoprotección: "Solo pasó una vez, mejor no lo cuento para no preocuparme ni preocupar a mi familia. Seguro que no vuelve a pasar" (testimonio de E.S. del 21 de enero de 2017). - Sentimiento de venganza, odio, culpabilización: "La culpa es mía por ser así" (testimonio de T.F. del 18 de enero de 2017).

- Desconfianza social ya que predomina la impunidad frente al apoyo y reconocimiento: "Nada va a cambiar" (testimonio de L.G. del 21 de enero de 2017).

- Aislamiento social para no molestar, no tener que recordar o no reexperimentar: "Si me quedo en casa nadie tendrá que preocuparse por mí" (testimonio de C.R. del 18 de enero de 2017).

Tabla 3: Impactos de las violencias sobre las mujeres leídas como discapacitadas.

la organización colectiva de las mujeres leídas como discapacitadas para denunciar las violencias a las que se ven sometidas y reivindicar sus derechos, perpetuándose la estigmatización social (ocultamiento de la problemática a nivel social) y una condiciones de vida con escasas o nulas oportunidades (educativas, sanitarias, laborales, etc.)

\section{Fragmentación de la comunidad}

La permanencia de un modelo social basado en la estratificación alimenta un sustrato de violencia que puede ser aplicado sobre cualquier persona que sea leída como diferente en un contexto carente de equilibrio social y que no reconoce la diversidad que existe en su seno. La tabla 4 muestra las consecuencias de este modelo sobre los vínculos sociales.

Las relaciones de apoyo para hacer frente a estos impactos, como decíamos, no se dan en el vacío, sino en un contexto ideológico determinante de los modelos de ayuda o solidaridad que pueden promover la dignidad de las mujeres o, por el contrario, dañar su identidad y limitar su autonomía. Si en la definición de discapacidad, si en el diseño de políticas y programas o si en el análisis de los impactos no se incorpora la perspectiva psicosocial, las formas de abordarla seguirán siendo asistenciales, promotoras de dependencias y perpetuadoras de diferentes formas de violencias.

\section{Qué es la diversidad funcional}

Diversidad funcional es un término acuñado en el marco del Modelo de Vida Independiente ${ }^{6}$, del que se nutre

6 El Movimiento de Vida Independiente es un movimiento social que nació en el marco de la lucha por los derechos civiles de finales de los años 60 en los Estados Unidos (Universidad de Berkeley). El movimiento fue impulsado por la acción de un grupo de personas con DF que necesitaban asistencia personal para realizar sus actividades diarias. La lucha se realizó desde el Modelo de la Diversidad y que viene a desplazar a los anteriores modelos empleados en el ámbito de las personas leídas como discapacitadas que promueven la dependencia, el asistencialismo, la institucionalización, la rehabilitación y medicalización de las personas leídas como discapacitadas. El Modelo de la Diversidad se basa en los principios de los Derechos Humanos: dignidad, diversidad, igualdad de derechos e igualdad de valor de las vidas, leídas como discapacitadas o no, entendiendo que existen diferentes formas de ser y de "funcionar" en la sociedad. La tabla 5 muestra las características del concepto de DF para las mujeres del grupo motor.

Como ya hemos mencionado, el término diversidad funcional no es un sinónimo de discapacidad, no es un eufemismo ni un término políticamente correcto para referirnos a un grupo de la sociedad tradicionalmente discriminado y excluido, al que se ha considerado menos capaz. El concepto de DF pone de relieve las diferentes capacidades que tenemos todas las personas de la sociedad y cómo todas necesitamos de las otras para funcionar y hacemos uso de diferentes apoyos, técnicos o personales, para desenvolvernos en el día a día. Algunos ejemplos los podemos encontrar en el uso de gafas, lentillas, alfabeto Braille o asistentes personales para mirar o leer. Otros los encontramos en las maneras de caminar (ya sea con bastón, andador o silla de ruedas) o de comer (con cubiertos que sostiene la propia persona u otra externa que le apoya). A todo esto es a lo que llamamos interdependencia, la relación recíproca natural, en la que todas las personas

las organizaciones civiles, universidades y asociaciones de veteranos de guerra. Su objetivo era que las personas discriminadas por su DF pudieran salir de los hospitales e instituciones e incluso de sus casas, en donde se hallaban recluidas, para que pudieran vivir y participar en la comunidad. Este movimiento llega a España en el año 2001, a través del Foro de Vida Independiente y Divertad (FVID) (Foro de Vida Independiente, 2018). 


\section{Impactos comunitarios}

- Alteración de las formas de convivencia y debilitamiento de las redes sociales.

- Homogeneización de los valores culturales: pérdida de referentes enriquecedores, estandarización del modo de vida, etc.

- Sostenimiento del estigma político: minusvaloración de los efectos de la discapacidad (naturalización de la violencia) y limitación de los derechos.

- Polarización social: los normales y los anormales, los capacitados y los no capacitados.

- Cohesión grupal frente a un enemigo, inventado o construido socialmente: se percibe que las personas leídas como discapacitadas se llevan más ayudas que el resto o que por su culpa hay personas que no pueden tener una vida normal.

- Evitación, rechazo, resignación y escepticismo ante las posibilidades de cambio, como clima social mayoritario.

- Las personas más afectadas tienen menos apoyo. No se produce un cambio o transformación social de las situaciones de exclusión, aislamiento o marginación.

- Predominancia de un modelo de participación de carácter manipulativo, simbólico, instrumental o por incentivos, para simular un cambio en el modelo.

- Los modelos de relación de ayuda o apoyo social se fundamentan en la idea de asistencia o caridad.

Tabla 4: Impactos de las violencias a nivel comunitario.

\section{¿Qué es la diversidad funcional? (una definición desde el proyecto “Miradas")}

Un término:

- Positivo, que dignifica y reconoce las diferencias individuales de cada persona.

- Que atribuye las limitaciones funcionales y/o barreras (arquitectónicas, emocionales, comunicativas, etc.) a la estructura social.

- Inclusivo, que incorpora a todas las personas con sus diferentes formas de funcionar, eliminando así la discriminación ejercida hacia un sector de la población.

- Que promueve las capacidades, oportunidades y deseos.

- Que favorece la (inter)dependencia de las personas, evitando la segregación, el aislamiento, la soledad...

- Que supone un reconocimiento y garantía de derechos sociales.

- Que favorece la aplicación del diseño universal, atendiendo a nuestras necesidades, teniendo en cuenta todas las formas de funcionar y las diferentes etapas de nuestra vida.

- Y que asegura y amplía nuestra autonomía, autodeterminación y capacidad de influencia social.

Tabla 5: ¿Qué es la diversidad funcional?

necesitamos de las otras para la supervivencia y el desarrollo eligiendo, de entre los diferentes recursos que tenemos a nuestra disposición, aquellos que nos generan bienestar social y emocional en nuestra vida diaria. Sin embargo, y como consecuencia de la estandarización de la sociedad, hay recursos que se consideran válidos y de ahí que las personas que los usan están dentro de la curva de la normalidad (aquellas leídas como normales), y otros que son considerados especiales, que convierten a las personas usuarias de los mismos en sujetos especiales, alejándoles o distanciándoles aún más de esa curva de la normalidad y por tanto del goce de la mayoría de derechos sociales (Rodríguez y Ferreira, 2010).

El término diversidad funcional, por tanto, puede entenderse "como un fenómeno, hecho o característica presente en la sociedad que, por definición, afectaría a todos los miembros por igual, (). Es decir, dado que en la sociedad existen personas con capacidades o funcionalidades diversas o diferentes entre sí, incluso dentro del mismo individuo con diferentes edades, afirmaríamos que, en un momento dado, en la sociedad (o en un determinado grupo social) existe o se produce diversidad funcional (del mismo modo que se observa diversidad cultural, sexual, generacional...)" (Mirwald, 2016).

En una situación ideal en la que la discapacidad, como concepto o barrera, no existiera, ni siquiera nos haría falta hacer uso de ningún término específico para referirnos a unas personas u otras, ya que la diversidad estaría aceptada, incluida e interiorizada en nuestras formas de hacer $y$ pensar, y no sería algo especial o diferente. Pero, mientras sigan existiendo esas barreras, limitaciones y etiquetas que estigmatizan y generan situaciones de multidiscriminación, apostamos por utilizar el término "mujeres leídas como discapacitadas" como forma de visibilizar esas violencias y poner en el centro del debate público su pervivencia y las numerosas discriminaciones o desigualdades a las que se ven sometidas muchas mujeres que, lejos de ser también diversas funcionales, ven como su identidad personal y social se construye desde una represión que es acumulativa en función de una cadena de intersecciones (Crenshaw, 2002): clase social, edad, raza, orientación ideológica y religiosa, orientación sexual, entre otras.

Siendo conscientes de la trayectoria de trabajo del Movimiento de Vida Independiente y de la importancia que para muchas personas y colectivos tiene la lucha por la dignidad, entendemos el término DF como una herramienta para transitar hacia una sociedad diseñada, en todos los sentidos, para la diversidad. Una diversidad en la que todas las personas estaríamos incluidas, dejando atrás las desigualdades y discriminaciones de las que hemos hablado en este artículo. Una sociedad libre de categorías y estigmas. Nuestra intención es reclamar, junto a ellas y a ellos, el diseño y la implantación de un conjunto de medidas de acción positiva que así lo permitan. 
Violencias contra las mujeres leídas como discapacitadas. Multidiscriminación e interseccionalidad

La violencia no es un impulso basado únicamente en factores biológicos. Esta se produce en el marco de un contexto social, cultural y político concreto, lo que determina sus características e intensidad. Su intencionalidad está vinculada al modelo de estratificación u ordenación social en el que están insertas las personas. El conjunto de discriminaciones a las que las mujeres leídas como discapacitadas son sometidas permite mantener el estatus social de otras muchas personas erróneamente llamadas "normales" (Rodríguez y Ferreira, 2010). Esto es lo que hace que las violencias se puedan mantener y formen parte de la base estructural de la sociedad.

En concreto, la violencia contra las mujeres es una violación de los derechos humanos que se ejerce sobre todas las mujeres del mundo, manifestada de diferentes formas dependiendo del contexto (INWWD, 2010). No existe un perfil único de hombre que ejerce violencia, ni de mujer que la sufre, siendo muchos de los aspectos de esa violencia de carácter universal. La violencia contra las mujeres se fundamenta en las relaciones de poder y desigualdades entre hombres y mujeres y, se puede dar en el ámbito familiar, escolar, laboral, público e institucional. La naturalización con la que la sociedad trata la desigualdad que organiza los roles de género, hace que las violencias derivadas del sistema patriarcal sean invisibles o difíciles de detectar en la mayor parte de las ocasiones.

El género como paradigma o como categoría analítica resulta de suma importancia a la hora de identificar la influencia del patriarcado como factor estructural donde se imprimen las pautas de dominación masculina, de cara a comprender la diversidad de violencias que afectan a las mujeres leídas o no como discapacitadas (Michelle y Adrianne, 1998). No se pueden excluir otros elementos que forman parte de la identidad (múltiple) de cada persona en un contexto concreto. De esta manera, el análisis interseccional ${ }^{7}$ revela que factores como la clase socioeconómica, la cultura, la orientación sexual, la ascendencia, la capacidad, la localización geográfica, entre otros, al cruzarse con el género, se convierten en experiencias múltiples de opresión y discriminación (clasismo, racismo, heterosexismo, capacitismo, etc.).

Considerar, además del género, otras desigualdades, exige pasar de un enfoque unitario a un enfoque que ha de integrar las desigualdades múltiples. La dimensión de género, como factor (no causa) generador de situaciones de discriminación y violencia, es un desencadenante más, entendiendo éste como aquel que necesita de unas condiciones previas externas para poder derivar en esa acción, y que lleva a muchas mujeres a una situación de múltiple riesgo, opresiones entrelazadas o multidiscriminación (Crenshaw, 2002), que tiene como consecuencia la exposición de las mismas a situaciones de alto impacto psicosocial y a una configuración de la identidad social y personal basada en una relación de subordinación e inhabilitación.

7 La idea de intersección fue introducida por Kimberle Crenshaw para visibilizar la existencia de varios ejes de desigualdad (raza, etnia, género, etc.) que aunque operan de manera independiente, cuentan con varias intersecciones entre ellas (Crenshaw, 2002).
Así, lo anterior evidencia que la violencia no se agota sólo en ser leída como mujer y como discapacitada, sino que además existen otras categorías asignadas que, al cruzarse, suponen un mayor número de violencias. Al aplicar el enfoque de la interseccionalidad en el análisis de las violencias que sufren las mujeres leídas como discapacitadas descubrimos que se ven sometidas a un número mayor de violencias naturalizadas e invisibilizadas socialmente, que no se conocen, no interesan y que, como consecuencia, no se les presta atención (Platero, 2002).

$\mathrm{Si}$ entendemos el término discapacidad como el conjunto de barreras impuestas por la sociedad por el hecho de no responder a una norma social (Díaz, 2009), la discapacidad es un ejercicio de violencia al que se somete de manera permanente a las personas leídas como discapacitadas. Podemos decir, por tanto, que la violencia genera discapacidad y agrava la imposibilidad de salir de la misma. Si además entendemos la discapacidad como una categoría social que permite que las personas sean desacreditadas socialmente, la discapacidad, al igual que veíamos con el género, es un factor desencadenante de situaciones de violencia, pero en ningún caso podemos tratarla como una causa. Siguiendo con la propuesta del enfoque de la interseccionalidad, la discapacidad es una excusa o camino sobre el que transitan las violencias. En el caso de las mujeres leídas como discapacitadas, esas condiciones previas son las relaciones de poder desiguales entre hombres y mujeres, generadoras de situaciones de discriminación que detallamos a continuación.

La primera discriminación que encontramos tiene que ver con la universalización o estandarización de los géneros, de tal modo que existe un tipo de mujer socialmente establecido al que se hace referencia cuando se habla de la mujer (García-Santesmases, 2015). Ese tipo es: blanca, occidental, heterosexual, sin discapacidad, de mediana edad, de clase media o baja. Esta construcción de género en torno a un cuerpo y a unas identidades sociales concretas es tan efectiva que muchos tipos de violencias machistas sobre muchos tipos de mujeres se quedan en los márgenes de lo socialmente aceptado, de la composición de los colectivos o grupos de apoyo mutuo, de los programas de atención y prevención de las violencias machistas o de las campañas de sensibilización, por poner sólo algunos ejemplos. La creación de este tipo de mujer también aparece en el ámbito de la diversidad funcional.

Al tratarse de una categoría social y afrontarse de manera global, cuando se habla de mujeres con discapacidad el referente normal es: mujer con discapacidad física, blanca, occidental, heterosexual, de clase media, de mediana edad y de tradición judeocristiana. Esta homogeneización, como en el caso anterior, no sólo mantiene las estructuras y dinámicas sociales que perpetúan las violencias machistas sobre mujeres que son diversas, sino que también reduce las posibilidades de identificación de dichas violencias y, con ello, la creación de medidas concretas y efectivas para su erradicación, prevención o intervención.

Otra discriminación básica es el capacitismo o la creencia de que algunas personas no responden a la norma de productividad desde el punto de vista economicista porque sus cuerpos, al no ser normales, no son capaces o productivos, y por tanto son menos valiosos, siendo objeto permanente 
de cura, rehabilitación o de una mayor protección. Como apuntábamos anteriormente, todo esto conlleva una amplia terminología utilizada a la hora de nombrar a las personas leídas como discapacitadas: enfermas, minusválidas, discapacitadas, deficientes, invidentes, anormales, retrasadas, etc. La discriminación socava el poder sobre sus propias vidas, genera aislamiento y subordinación, refuerza la espiral de dependencia, indefensión, vulnerabilidad, incapacidad, en definitiva, genera la interiorización de los atributos propios de su estigma.

Este falso consenso social sobre lo que es normal genera un buen número de juicios de valor sobre lo que es aceptable o correcto. Si lo aceptable o esperado para una mujer es ser deseable y orientarse hacia los cuidados, entre algunas cosas, las mujeres leídas como discapacitadas van a encontrar un mayor número de barreras a la hora de relacionarse y comunicarse, basadas en el aislamiento, el menosprecio, los malos tratos y las violencias machistas, ya que no responden a ese patrón de belleza homogeneizado ni a las expectativas de rol esperadas. Se les considera un "fallo", con cuerpos "enfermos", seres defectuosos, incompletos, incapaces, sin emociones o sentimientos que se puedan regular. De esta manera, las mujeres leídas como discapacitadas, al igual que otros grupos sociales, son percibidas como desacreditables (Goffman, 2012) y, por tanto, desacreditadas en las relaciones sentimentales y en todas las interacciones sociales. En el escenario social, tanto público como privado, se puede abusar de ellas sin pudor, ya que no se percibe como algo negativo o doloroso; además se puede hacer con impunidad, ya que aquello que se sale de la "curva de la normalidad", puede merecer ese tipo de tratamiento, violencia o desacreditación.

Una discriminación más es el modelo dominante de sexualidad, que orienta el deseo hacia la norma heterosexual y la práctica genital desde un enfoque médico preventivo. De esta manera, las mujeres leídas como discapacitadas reciben una mayor sobreprotección tanto en el entorno familiar como en el institucional, siendo percibidas como sujetos de una mayor o especial vulnerabilidad relacional o afectiva, porque se piensa que no entienden qué es el amor o que el amor les puede hacer más daño; se piensa que hay más riesgo de embarazo o que no pueden llegar a tener y mantener una pareja, ni ser madres, entre otras cosas. Esta infantilización supone una negación del desarrollo afectivo-sexual de manera plena, ya que es difícil encontrar en las familias una especial atención a la educación en sexualidades, pero mucho más difícil es encontrarla en los centros y recursos que frecuentan las mujeres leídas como discapacitadas.

Es importante señalar que gran parte de las acciones de educación sexual promovidas desde las familias, las asociaciones y las instituciones tienden, desde un punto de vista coital, a asexualizar o hipersexualizar a las mujeres leídas como discapacitadas, operando sobre los cuerpos, como decíamos, para protegerlos o infantilizarlos, reduciendo la intimidad, las posibilidades de establecer relaciones afectivas horizontales y la autonomía sexual. Tampoco se tiende a hablar de homosexualidad o transexualidad en este ámbito, por lo que no se visibiliza que la diversidad también es sexual. Las consecuencias de esto no son sólo la represión o inhibición de deseos y fantasías (falta de reconocimiento social del erotismo de los cuerpos no normativos), sino también el ser víctimas de abusos en los que las personas agresoras salen impunes, lo que hace que sean más vulnerables y dependientes.

Las consecuencias van en línea con las anteriores: desarrollo de una mayor percepción de vulnerabilidad, situaciones objetivas de dependencia emocional, física y económica; pérdida de la titularidad de derechos civiles, "devaluación social" (por no responder a las expectativas de género) y deterioro de las habilidades, estrategias o recursos de afrontamiento ante las violencias machistas tanto a nivel individual como colectivo. En definitiva, las mujeres son consideradas "ciudadanas de segunda o de tercera" al ser relegadas a grupos sociales no productivos y tratadas como "clientes pasivos" de programas de ayuda que, supuestamente, requieren de una especial inversión de recursos o implican un gasto desmesurado ${ }^{8}$.

Como contábamos anteriormente, algunas de las manifestaciones de las violencias machistas no son visibles o identificadas como violencias debido a que han sido normalizadas por la sociedad, aceptadas y legitimadas por los estamentos judiciales y la administración, aludiendo a la pertinencia y adecuación de las medidas tomadas. "Es lo mejor para ella" es un argumento al que de manera recurrente las mujeres se siguen enfrentando.

Algunos ejemplos que hemos identificado han sido: la esterilización sin consentimiento como forma de infantilización y negación del derecho de las mujeres leídas como discapacitadas a la maternidad, intervenciones e institucionalizaciones psiquiátricas forzadas debido a comportamientos desajustados, constantes negligencias médicas por desinterés o la falta de formación entre las y los profesionales, inexistencia de material técnico adaptado porque la medicina no se piensa desde la diversidad de los cuerpos y la negación del desarrollo personal o profesional porque para la sociedad no resultan funcionales o productivas. Todos estos, entre otros, son actos de violencia que se llevan a cabo dentro del marco legislativo de la política estatal y que dificultan la detección de otras formas de multidiscriminación.

También encontramos otras muchas formas de violencias que se ejercen en el ámbito familiar, donde se contabiliza el mayor número de personas cuidadoras, y en el que en muchas ocasiones se sobreprotege, infantiliza y se priva de cualquier tipo de autonomía o identidad y por lo tanto de la toma de decisiones o del ejercicio de la autodeterminación.

Por último, los centros y recursos socio-asistenciales juegan un papel muy importante en el ejercicio de violencias ya que se dan porcentajes altos de abusos por parte de las personas cuidadoras y de parte del equipo de profesionales. Como en los otros ámbitos, estas discriminaciones son normalizadas, invisibilizadas, silenciadas y encubiertas por las propias administraciones, responsables del control y la supervisión de estos centros. Junto a las violencias familiares, esta situación agrava la falta de posibilidades de desarrollo de las mujeres y genera una situación de desconfianza e indefensión basada en la percepción de que no existen

8 Se puede ampliar la información sobre las especificaciones de los malos tratos hacia mujeres leídas como discapacitadas en el informe Violencia y mujer con discapacidad elaborado por la Asociación Iniciativas y Estudios Sociales (1998) pubiclado por Iglesias et al. 
posibilidades de control o afrontamiento que permitan salir de la violencia.

Como puede verse en estos primeros ejemplos, las violencias de las que hablamos se dan en todos los ámbitos de la vida, se manifiestan de forma diferente con patrones repetitivos y responden a esa estructura social patriarcal y capacitista de la que hablábamos al principio del artículo. Las diferentes técnicas de diagnóstico utilizadas a lo largo del proyecto nos han permitido detectar y sistematizar algunas de estas violencias que presentamos a continuación a través de sus testimonios.

- Violencia física. Engloba todas aquellas acciones directas o indirectas que ponen en riesgo el bienestar físico, la vida y la salud, provocando dolor, sufrimiento o lesiones; además de la negación o privación de necesidades básicas para el mantenimiento de la salud, la higiene y la apariencia: "Me administraban anticonceptivos en la comida sin mi consentimiento" (testimonio de G.D 18 de enero de 2017); "A pesar de que yo era independiente en la higiene corporal, no me permitían ducharme sola y a mi ritmo aludiendo a que era lenta, por lo que no tenía intimidad" (testimonio de S.B. 18 de enero de 2017); "En la consulta ginecológica no tenían material ginecológico para cuerpos no normativos así que las revisiones no se llevaban a cabo, a pesar de mis continuas visitas por dolores. Cuando se quisieron dar cuenta tenía cáncer de útero y me hicieron una histerectomía, algo que se podría haber evitado"(testimonio de C.R. 18 de enero de 2017).

- Violencia emocional. Son las acciones que dañan el bienestar y el equilibro emocional de la mujer; incluyendo actos de privación o negación de la atención: "La gente se burla de ti cuando te lee como discapacitada. Me ha pasado a lo largo de mis 50 años, en los centros, en la calle $Y$ eso me hace sentir mal y triste" (testimonio de T.F. 21 de diciembre de 2016); “El primer día de mi regla la reacción de mi padre fue infravalorarme como persona y como mujer, haciendo comentarios horribles. Pensaba que yo era inútil y por lo tanto que tuviera la regla también" (testimonio de L.G. 18 de enero de 2017); "A veces la gente habla delante de mí y sobre mí, como si yo no existiera, como si no me enterara, y preguntan sobre mí a mi madre cuestiones personales, algo que nunca harían si no me leyeran como discapacitada" (testimonio de E.S. 21 de diciembre de 2016); "Cuando dije que quería casarme mi madre se cabreó muchísimo porque pensaba que iba a tener que llevar dos casas, la suya y la mía con mi pareja" (testimonio de L.G. 18 de enero de 2017); "Quisimos ir a uno de los centros de día de la red de violencia de la Comunidad de Madrid para conocerlo y encontrarnos con uno de los grupos de mujeres que se reúnen allí. El ascensor no era lo suficientemente ancho, por intérprete de lengua de signos ya ni preguntamos" (testimonio de G.D. 25 de enero de 2017).

- Violencia sexual. Son todas aquellas acciones relacionadas con el ámbito de la sexualidad que no sean consentidas, generando así un daño emocional y/o físico en las mujeres: "Desde que tenía siete años un amigo de la familia me tocaba. Me sentía incómoda pero no sabía que estaba mal. Un día se lo conté a mi familia y nunca más volví a ver a ese hombre. Hasta que no he sido bastante mayor no supe que había sufrido abusos sexuales. Nunca nadie me explicó nada" (testimonio de C.P. 18 de enero de 2017);
“Entré en el portal de mi casa y detrás entró un hombre que decía ir a ver a un amigo. Cuando me di cuenta estaba pegado a mi espalda con el pene en la mano" (testimonio de M.L. 21 de diciembre de 2016); "Caminando por la calle un hombre se acercó y me dijo cosas asquerosas, le mandé a la mierda y su reacción fue decirme que me iba a violar por bocazas" (testimonio de S.B.21 de diciembre de 2016); "Cuando no quería tener relaciones con mi marido, él me quitaba las muletas sabiendo que yo no me podía mover y me dejaba inmovilizada en la cama. Sólo me las devolvía después de violarme" (testimonio de A.V. 21 de diciembre de 2016); "No me dejan maquillarme o ponerme ropa con la que me siento a gusto" (testimonio de C.P. 11 de enero de 2017); "Me gustaría comprarme un vibrador pero no cuento con una persona de confianza para que me lo compre. Si se lo digo a mi familia se asustaría" (testimonio de C.P. 25 de enero de 2017); "Cada vez que voy a comprarme ropa, me dicen que no hay ropa para mí" (testimonio de T.R. 18 de enero de 2017).

- Violencia económica. Son todas las acciones que supongan la privación de derechos y control sobre las propiedades, ganancias propias y comunes, así como el uso de la imagen de la mujer por parte de terceros para realizar actividades que supongan un lucro para terceras personas: "No me dejaban estudiar, trabajar o salir sola a la calle, sin embargo me he ocupado siempre de las tareas del hogar, la cocina y mis tres hermanos menores" (testimonio de T.R. 11 de enero de 2017); "En el centro siempre te amenazan con la asignación económica semanal, o haces lo que quieren o te quedas sin dinero" (testimonio de G.D. 21 de diciembre de 2017); "Tanto la pensión como la indemnización que me asignaron por el accidente, siempre han estado gestionadas por mi familia, nunca he visto un euro y sin embargo tengo que rogar para poder tener algo de ese dinero" (testimonio de C.P. 21 de diciembre de 2017); "Estoy todo el año trabajando en el centro ocupacional para ganar un dinerillo para las vacaciones con la asociación y cuando llega este momento no me dejan gestionar mis propios ahorros, se lo dan a las responsables del viaje" (testimonio de S.B. 21 de diciembre de 2017).

- Violencia institucional. Es la ausencia de actuaciones, por parte de las administraciones, contra las violencias anteriores, que favorecen la perpetuación de las mismas sobre las mujeres con diversidad funcional y generan en éstas un daño físico y emocional, además de exponerlas a una situación real de abandono y desprotección (INWWD, 2010). Algunos ejemplos identificados a lo largo del proyecto han sido: limitación de acceso a recursos generales, negación de la identidad no estigmatizada, limitación en la participación de la toma de decisiones institucionales sobre su capacidad, negación de los derechos sexuales y reproductivos (esterilización sin consentimiento, administración de anticonceptivos hormonales sin consentimiento o por medio de engaño, aborto forzoso, etc.), falta de formación de los y las profesionales de los servicios públicos, ausencia de material técnico adaptado para las supervisiones médicas, no investigación ni persecución de los abusos dentro de las instituciones, institucionalización forzosa, falta de accesibilidad en los servicios públicos (barreras arquitectónicas, comunicativas y actitudinales), negación del ejercicio de los derechos en igualdad de condiciones, 
paralización de las leyes específicas y de medidas de acción positiva por falta de recursos económicos, imposibilidad de acceso a los recursos de la red de violencia de género o sexual y limitación en la información o información sesgada.

\section{CONCLUSIONES}

A lo largo de este artículo, hemos visto cómo las mujeres leídas como discapacitadas sufren una serie de violencias específicas por el hecho de ser leídas como mujeres y como discapacitadas, por vivir en un entorno que no ha sido diseñado, arquitectónica ni socialmente para acoger todas las diversidades.

No es lo mismo ser víctima en una sociedad que reconoce y apoya, que en una sociedad que señala a las mujeres leídas como discapacitadas como culpables de dicha discriminación y que las aísla o rechaza cuando intentan salir de esta situación. Desde este enfoque, los modos de ayuda o superación del impacto de la discapacidad no pueden reducirse a lo asistencial. Todo lo contrario, han de partir de la situación de la persona, orientarse hacia la reducción de las causas sociales, ofrecer espacios comunitarios de apoyo mutuo, compartir colectivamente formas de afrontamiento o diseñar mecanismos de reconocimiento, reparación y no repetición, entre otras actuaciones. Cada vez es más necesaria la puesta en marcha de un nuevo modelo social, el de la diversidad. Un modelo con el que podemos invertir el modelo de normalidad y funcionalidad que sostiene la estructura heteropatriarcal y que beneficia de manera equitativa a todos los estratos de la sociedad.
Desde el proyecto Miradas (Ojosqueoyen y Andecha, 2017) elaboramos junto a otros actores sociales una propuesta de trabajo para el futuro en clave de estrategias de diferente tipo.

En primer lugar, estrategias individuales, ya que, como decíamos, no todas las mujeres responden de la misma manera ante las violencias. Las estrategias que presentamos en la tabla 6 ejemplifican diversas maneras de afrontar $y$ resistir para mantener su integridad.

En segundo lugar, estrategias colectivas dado que, a pesar de que el apoyo social es muy beneficioso para enfrentar violencias y multidiscriminación, las mujeres leídas como discapacitadas tienen muchos problemas para obtenerlo. Si la mayor parte de las barreras o dificultades que afrontan tienen que ver con la falta de una respuesta social ante el estigma de la discapacidad, es fácil encontrar que las estrategias individuales no suelen ir acompañadas de otras de carácter colectivo, o solo se producen de manera puntual o descoordinadas de otras, minimizando su efecto.

Desde el proyecto Miradas, consideramos que es necesario generar estrategias colectivas basadas en el apoyo mutuo y la creación de redes, que permitan visibilizar las situaciones de violencia y multidiscriminación a las que están expuestas, y promover su organización grupal, al igual que el resto de colectivos oprimidos, para dar una respuesta contundente ante las mismas y exigir la asunción de responsabilidades tanto a nivel social como institucional. Por tanto, las estrategias institucionales no pueden faltar. Si a la hora de analizar el impacto de las violencias es importante hacerlo desde un enfoque psicosocial, en el diseño de

\section{Experiencias de afrontamiento individual}

- Hacer justo lo contrario a lo que me dicen o recomiendan. Ante instrucciones o mensajes del tipo: "no eres capaz", "no lo intentes", no hacer caso y poner en valor tus propios deseos.

- Ser sincera contigo misma y coherente con los propios deseos, creencias, etc.

- Vivir comprometida con tus ideas.

- Mantener la alegría y una visión positiva de una misma. Atender las propias necesidades y poner en marcha actitudes de autocuidado.

- Tener capacidad para aceptar la realidad, no en términos de conformismo, sino aceptando con realismo las situaciones duras que a veces ofrece la vida.

- Tener confianza en mí misma, no ponerse en duda y no dejarse llevar por las devoluciones negativas que hace la gente fruto de los estereotipos y el desconocimiento.

- Buscar e intentar encontrar otro tipo de referentes, más positivos o más acordes a los propios deseos y objetivos para ganar en percepción de control y seguridad.

- Salir del entorno que genera o perpetua la dependencia.

- Ampliar la red de apoyo para no depender o hacer dependientes siempre a las mismas personas.

- Reapropiarse de la burla recibida y usar la ironía o el humor: por ejemplo, probarte la ropa en la entrada del comercio al que no puedes entrar porque no es accesible.

- Ante situaciones de inaccesibilidad en el metro (ascensor roto o que la silla de ruedas se quede enganchada al entrar al vagón), intentar aceptar la situación y llevarlo lo mejor posible, compartiendo las dificultades que plantea a tu entorno. Buscar apoyo para poner una queja o denuncia.

- Poner en marcha estrategias de visibilización de la queja ante situaciones que se perciben como injustas o que atentan contra la autonomía y autodeterminación: poner pancartas, hacer huelgas simbólicas, etc.

- Analizar la situación para valorar la respuesta cuyo coste emocional sea el más asumible para una misma.

- Promover el conocimiento público o a nivel social de la experiencia personal o vivencia de la discapacidad y de la diversidad funcional, para promover la empatía.

- Ser persistente en tus objetivos, sin hacer caso de los comentarios limitantes.

- Mantener la firmeza ideológica. Evitar situaciones de victimismo y pensarse como una mujer en proceso de lucha. 


\section{Acciones estratégicas de carácter institucional}

- Dotación de presupuesto a la legislación vigente para su correcta implementación.

- Aplicar el enfoque de derechos humanos, de diversidad y de género a todas las políticas públicas de manera transversal.

- Seguimiento de la normativa, investigación de la aplicación de la misma y sanciones ejemplares para el incumplimiento.

- Incluir a las mujeres leídas como discapacitadas y mujeres con diversidad funcional, en el diseño de las políticas públicas a través de procesos comunitarios, reuniones de expertas, organizaciones independientes, etc.

- Diseño universal de las políticas públicas y recursos dotacionales, desde la perspectiva de género y de diversidad, haciendo especial hincapié en la accesibilidad (arquitectónica, comunicativa, emocional) de los recursos existentes de la red de violencia de género de las diferentes administraciones públicas (municipales, regionales y estatales).

- Universalización de la formación en género, diversidad y violencias, haciendo especial énfasis en la sensibilización y capacitación de las personas profesionales de la red de recursos públicos y privados, que contemplan dentro de sus responsabilidades la atención al público en general.

- Puesta en marcha de medidas de sensibilización en diversidad funcional y de promoción de la vida independiente.

- Diseño y ejecución de programas de acompañamiento y apoyo psicosocial para las familias, los menores y las mujeres.

- Investigación en materia de violencias contra las mujeres leídas como discapacitadas. Presentación de datos desagregados. - Universalización de la formación e información sobre derechos fundamentales, sexualidad, autonomía y autodeterminación, género y multidiscriminación hacia las mujeres leídas como discapacitadas y su entorno social.

Tabla 7: Recomendaciones para el abordaje de las violencias a nivel institucional.

estrategias de afrontamiento es importante promover la interrelación de diferentes actores, con especial énfasis en los institucionales. Su papel es fundamental a la hora de garantizar el cumplimiento de los derechos fundamentales y garantizar medidas que eliminen las situaciones de multidiscriminación desde principios de no repetición. Eso implica el reconocimiento de un modelo de vida en sociedad basado en la desigualdad y la puesta en marcha de un modelo de gobierno basado en el fortalecimiento comunitario y el reconocimiento de la diversidad a partir de propuestas como las que se plantean en la tabla 7.

\section{AGRADECIMIENTOS}

El proyecto "Miradas: mujer diversidad funcional y multidiscriminación" fue implementado gracias a la colaboración de Andecha -Participación y Trabajo Comunitario-, Asociación OjosqueOyen, Obra Social Fundación La Caixa, Junta Municipal de Distrito Centro y Junta Municipal de Distrito Arganzuela del Ayuntamiento de Madrid y Asociación IPADEVI. Para su desarrollo contamos con la participación de los colectivos feministas Las Raras, Vecinas de Lavapiés, Bate Morado, y con las ponencias de María Naredo y Soledad Arnau. El proyecto no hubiera sido posible sin el grupo motor "Miradas" con ayuda de las cuales construimos nuestras miradas hacia las violencias machistas y la multidiscriminación sobre mujeres leídas como discapacitadas.

\section{REFERENCIAS}

Asociación OjosqueOyen y Andecha Participación y Trabajo Comunitario. 2017. Miradas: mujeres, diversidad funcional y multidiscriminación. Guía para la detección de las violencias contra las mujeres leídas como discapacitadas. Madrid: Asociación OjosqueOyen y Andecha Participación y Trabajo Comunitario.

Barnes, Colin. 2005. Independent Living. Politics and Policy in the United Kingdom: A Social Model Account. The Review of Disability Studies, 1(4): 5-13.
Crenshaw, Kimberlé. 2002. Documento para o encontro de especialistas em aspectos da discriminação racial relativos ao gênero. Revista Estudos Feministas, 10(1). http:// www.scielo.br/scielo.php?script=sci arttext\&pid=S0104$\underline{026 \times 2002000100011}$

Díaz, Eduardo. 2009. Reflexiones epistemológicas para una Sociología de la discapacidad. Intersticios: Revista Sociológica de Pensamiento Crítico, 3(2): 85-99.

Etxeberria, Xabier. 2008. La condición de ciudadanía de las personas con discapacidad intelectual. Bilbao: Universidad de Deusto.

Fine, Michelle y Asch, Adrienne (eds.). 1988. Women With Disabilities: Essays in Psychology, Culture and Politics. Philadelphia, PA: Temple University Press.

Foro de Vida Independiente. 2018. Consultado el 15/09/2018. http://forovidaindependiente.org/filosofia-de-vidaindependiente/

García-Santesmases, Andrea. 2015. El cuerpo en disputa: cuestionamientos a la identidad de género desde la diversidad funcional. Intersticios. Revista Sociológica de Pensamiento Crítico, 9(1): 41-62.

Goffman, Erving. 2012. Estigma. La identidad deteriorada. Madrid: Amorrortu.

Iglesias, Marita; Gil, Gema; Joneken, Anneli; Mickler, Barbel y Knudsen, Janne. 1998. Violencia y la mujer con discapacidad. Consultado el 14/10/2018. https://www. independentliving.org/docs1/iglesiasetal1998sp.html

INWWD (Red Internacional de Mujeres con Discapacidad). 2010. Violencia contra mujeres con discapacidad. Consultado el 14/10/2018. www.asoc-ies.org/violencia/ docs/INWWD-ViolenciacontraMujeresconDiscapacidad ESP.doc

Martín Beristain, Carlos y Riera, Francesc. 2003. Afirmación y resistencia: la comunidad como apoyo. Barcelona: Virus Editorial.

Mirwald, Walter. 2016. Diversidad funcional. Consultado el 14/10/2018. https://waltermirwald.files.wordpress. com/2016/10/diversidad-funcional.pdf

Oliver, Mike. 2008. Políticas sociales y discapacidad. Algunas consideraciones teóricas. En Barton, Len (ed.) Superar las 
barreras de la discapacidad, 19-32. Madrid: Morata.

ONU. 2005. Resolución de la Asamblea General de Naciones Unidas 60/147 Principios y directrices básicos sobre el derecho de las víctimas de violaciones manifiestas de las normas internacionales de derechos humanos y de violaciones graves del derecho internacional humanitario a interponer recursos y obtener reparaciones. https:// www.ohchr.org/sp/professionalinterest/pages/ remedyandreparation.aspx

Platero, Raquel L. 2002. Mujeres discapacitadas y malos tratos. Perfiles, 180: 14-15.

Rodríguez, Susana y Ferreira, Miguel. 2010. Desde la discapacidad hacia la diversidad funcional. Un ejercicio de dis-normalización. Revista Internacional de Sociología, 68(2): 289-309.

Romañach, Javier y Lobato, Manuel. 2005. Diversidad funcional, nuevo término para la lucha por la dignidad en la diversidad del ser humano. Foro de Vida Independiente y Diversidad. Consultado el 26/7/2018. http://forovidaindependiente.org/wp-content/uploads/ diversidad funcional.pdf

Sen, Amartya. 2000. Desarrollo y Libertad. Bogotá: Editorial Planeta.

Shum, Grace; Conde, Ángeles y Portillo, Inés. 2006. Mujer, discapacidad y violencia. El rostro oculto de la desigualdad. Madrid: Instituto de la Mujer.

Vargas, Laura; Bustillos, Graciela y Marfán, Miguel. 1995. Técnicas participativas para la educación popular. Madrid: Editorial Popular. 